\title{
About the safety of adjuvant vaccines in pregnant women: an expert opinion
}

\begin{abstract}
The problem of studying the safety of the use of adjuvant vaccines in pregnant women and methods for evaluating their immunological efficacy are discussed.
\end{abstract}

Keywords: pregnant women, adjuvant vaccines against influenza, safety, immunogenicity
Volume 3 Issue 2 - 2017

\author{
Kostinov MP, ${ }^{1,2}$ \\ 'Federal State-Funded Science Institution, Mechnikov Research \\ Institute of Vaccines and Sera, Russia \\ ${ }^{2}$ Federal State-Funded Educational Institution of Higher \\ Education, I.M. Sechenov First Moscow State Medical University \\ of the Ministry of Health of Russia, Russia
}

\begin{abstract}
Correspondence:Kostinov Mikhail Petrovich, Professor, Head of the Laboratory of Vaccine Prophylaxis and Immunotherapy of the Federal State-Funded Science Institution, Mechnikov Research Institute of Vaccines and Sera, Moscow, Russian Federation; Professor of the Department of Epidemiology Federal State-Funded Educational Institution of Higher Education, I.M. Sechenov First Moscow State Medical University of the Ministry of Health of Russia, Moscow, Russia, 105064, Moscow, Malyi Kazenyi pereulok, Russia, tel +7(495) 917-41-49, Email monolit.96@mail.ru
\end{abstract}

Received: December 02, 2017| Published: December 05,

2017

\section{Introduction}

The lessons of the last century's influenza pandemics made mankind to develop new vaccine manufacturing technologies that in short time after immunization should provide full protection against infection with minimal antigen consumption. In this regard in 2009-2010years clinicians began to use adjuvant vaccines against influenza with a reduced in 2-3times the number of influenza antigens. Adjuvant is a substance of various origin, which changes the dynamics of the development of immunity, accelerates and increases the level of postvaccinal antibodies and the duration of their preservation. Accordingly, depending on the adjuvant, its structure, changes the nature of the mechanisms of development of postvaccinal immunity which differs from the long-used split and subunit vaccines against influenza. Used more than 25years in pregnant women, they are safe and immunogenic. During the influenza pandemic in 2009-2010years not only split and subunit vaccines were used for the immunization of pregnant women, but also adjuvant vaccines. As indicated in numerous publications the immunization with adjuvant-containing preparation was accompanied by the formation of protective immunity without the development of serious adverse events.

It is known that the following clinical indicators are used to assess the clinical safety and immunogenicity of split and subunit vaccines for pregnant women: the incidence of local and systemic reactions, of respiratory infections and influenza, the growth and weight of the newborn, etc. But these parameters, as well as the conducted studies are insufficient for an introduction of adjuvant vaccines in the practice of obstetrician-gynecologists, neonatologists. The safety of adjuvant vaccines in pregnant women and their newborns should be confirmed not by theory, but by concrete research. In spite of the fact that the adjuvant vaccines are considered to be safe pharmaceutical preparation and are studied at the preclinical stage of the study on experimental animals, this should be confirmed in the I, II, III phases of clinical trials, not only by a general and biochemical analysis of blood, urine, but by the main parameters of the specific mechanisms of action of the vaccine adjuvant: the cellular immune response, the parameters of innate immunity, the cytokine profile, the hormonal profile of pregnant women, the parameters of development of the fetus and the function of their central nervous system, physiological adaptation of the newborn, level and duration of preservation of transplacental immunity in infants. In addition, the immunological effect of vaccination with adjuvant preparation of women depending on the trimester of pregnancy remains unexplained.

Of great importance are the methods used to evaluate the immunogenicity of adjuvanted vaccines, since the accepted criteria (CPMP) are based on the study of the humoral immune response only due to the use of split and subunit vaccines. However, adjuvant vaccines activate, except humoral, cellular immune response, and other mechanisms of post-vaccination immunity formation are involved. Perhaps the activation of the last one, in which memory cells are formed, is more significant than the synthesis only of specific antibodies, the protective property of which lasts within 1-1.5years. It also remains to be seen what level of post-vaccination antibodies should be considered to be protective for infants and young children, since generally accepted standards are based on the study of antibody levels for healthy adults. Perhaps the solution of this task together with obstetrician-gynecologists, neonatologists, the adoption of consensus will give a scientifically grounded answer and confidence in the introduction/creation of modern vaccines for the prevention of not only the flu but other infectious diseases. 


\section{Acknowledgements}

None.

\section{Conflict of interest}

Author declares that there is no conflict of interest. 